\title{
Scalable Video Transmission Over Rayleigh Fading Channels Using LDPC Codes
}

\author{
Manu Bansal ${ }^{a}$ and Lisimachos P. Kondi ${ }^{a}$ \\ ${ }^{a}$ Department of Electrical Engineering, \\ University at Buffalo, The State University of New York, \\ Buffalo, NY 14260
}

\begin{abstract}
In this paper, we investigate an important problem of efficiently utilizing the available resources for video transmission over wireless channels while maintaining a good decoded video quality and resilience to channel impairments. Our system consists of the video codec based on 3-D set partitioning in hierarchical trees (3-D SPIHT) algorithm and employs two different schemes using low-density parity check (LDPC) codes for channel error protection. The first method uses the serial concatenation of the constant-rate LDPC code and rate-compatible punctured convolutional (RCPC) codes. Cyclic redundancy check (CRC) is used to detect transmission errors. In the other scheme, we use the product code structure consisting of a constant rate LDPC/CRC code across the rows of the 'blocks' of source data and an erasure-correction systematic Reed-Solomon (RS) code as the column code. In both the schemes introduced here, we use fixed-length source packets protected with unequal forward error correction coding ensuring a strictly decreasing protection across the bitstream. A Rayleigh flat-fading channel with additive white Gaussian noise (AWGN) is modeled for the transmission.
\end{abstract}

The rate-distortion optimization algorithm is developed and carried out for the selection of source coding and channel coding rates using Lagrangian optimization. The experimental results demonstrate the effectiveness of this system under different wireless channel conditions and both the proposed methods (LDPC+RCPC/CRC and $\mathrm{RS}+\mathrm{LDPC} / \mathrm{CRC}$ ) outperform the more conventional schemes such as those employing RCPC/CRC.

Keywords: 3-D SPIHT, LDPC Codes, RS Codes, RCPC Codes, Product Code Structure, Unequal Error Protection, Wireless Video Transmission, Rate-Distortion Optimization

\section{INTRODUCTION}

In the recent past, there has been a tremendous increase in the capabilities of wireless multimedia devices and services. The demand to improve quality of such systems within the limited bandwidth resources motivates the interest in error-resilient multimedia coding methods. This research problem is best dealt by choosing, 1) a scalable source codec with good compression efficiency. A scalable bitstream can be produced by encoding the source only once for different quality requirements and can be decoded at different rates with progressive reconstruction quality, and, 2) an appropriate set of channel codes for efficient forward error correction (FEC).

Various methods for image and video transmission over wireless channels have been discussed in. ${ }^{1-4}$ Mostly, these are based on 2-D/3-D set partitioning in hierarchical trees (SPIHT) source $\operatorname{codec}^{5,6}$ and channel coding employing rate compatible punctured convolutional (RCPC) codes. ${ }^{7}$

In this paper, we propose a wireless transmission system for scalable video over flat-fading Rayleigh channel. The embedded 3-D SPIHT codec $^{6}$ is used for video coding and two different schemes for channel coding using, i) the serial concatenation of low-density parity check (LDPC) codes ${ }^{8,9}$ and RCPC/ cyclic redundancy check $(\mathrm{CRC}),{ }^{12}$ and, ii) the product code structure consisting of a constant rate LDPC/CRC code across the rows of the 'blocks' of source data and an erasure-correction systematic Reed-Solomon (RS) code as the column code are proposed. These channel coding methods provide unequal error protection (UEP) to the bitstream divided into packets of constant size. The problem of optimal resource allocation is then formulated and solved using Lagrangian multiplier method ${ }^{13-15}$ for the choice for different overall transmission rates. Performance of this

Correspondence E-mail: mbansal@buffalo.edu 
system is evaluated for different channel conditions and simulation results outperform those using the classical techniques.

The rest of the paper is organized as follows. Sections 2 and 3 give an overview of the source and channel codes, respectively, used in this work. Section 4 discusses the optimization techniques for efficient bit allocation for both the proposed channel coding techniques and Section 5, presents experimental results. Section 6 concludes the paper.

\section{EMBEDDED VIDEO CODING USING 3-D SPIHT}

3-D SPIHT 6 is a wavelet-based embedded video coder employing subband coding (SBC) technique. Basically, it is a 3-D extension of the highly successful 2-D SPIHT ${ }^{5}$ image codec. The underlying SPIHT algorithm is based on the concepts of first forming the spatial orientation trees, which are groups of wavelet transform coefficients organized into trees rooted in the lowest frequency or coarsest scale subband with offspring in several generations along the same spatial orientation in the higher frequency (resolution) subbands. This structure of a tree exploits the self-similarity and magnitude localization properties of the wavelet transformed image. It is assumed that if a coefficient magnitude in a certain node of a spatial orientation tree does not exceed a given threshold, it is very likely that none of its descendants will exceed that threshold. Further, the wavelet transform coefficients in these trees are tested against a magnitude threshold $2^{n}$, where $n$ is called the level of significance (i.e., bit-plane coding, starting with the most-significant bit) and partitioned into different sets according to their significance. Finally, the sign bits and refinement bits (for the coefficients that are tested to be significant earlier) are coded and transmitted for that value of $n$. The value of threshold is successively lowered by power of 2 and the process terminates when the desired rate or quality level is reached.

The basic algorithm for 3-D SPIHT is same as 2-D SPIHT, except that a 3-D spatial-temporal tree structure is formed here using the wavelet transform coefficients for a group of frames (GOF). Under certain conditions, 3-D SPIHT codec (even without motion-compensation) performs comparable to H.263 and outperforms MPEG2. As mentioned earlier, 3-D SPIHT codec produces an embedded bitstream which means that every lower rate bitstream is a prefix of higher rate bitstreams and hence, is totally rate scalable. This helps in progressive transmission of the bitstream. It also provides multiresolution scalability, precise rate control and low complexity that makes it an attractive codec for wireless transmissions.

\section{CHANNEL MODEL AND CODING}

A flat-fading Rayleigh channel with additive white gaussian noise (AWGN) and perfect interleaving (i.e., we assume that the samples of the Rayleigh random process $\alpha$ are independent identically distributed (i.i.d.)) is modeled for the transmission.

$$
r(t)=\alpha(t) s(t)+n(t)
$$

where $r(t)$ is the received signal, $s(t)$ is the transmitted signal, $\alpha(t)$ is a Rayleigh distributed random process and $n(t)$ is the AWGN.

\subsection{Forward Error Correction}

While 3-D SPIHT codec gives a very good compression efficiency, due to it's embedded nature, even a single bit error can lead to the unrecoverable video at the decoder. When transmitting video over an unreliable channel, channel coding in the form of FEC is needed for error detection and correction. As mentioned earlier, we have used a two channel coding techniques employing, i) the serial concatenation of LDPC $\operatorname{codes}^{8,9}$ and RCPC $\operatorname{codes}^{7} /$ CRC code, ${ }^{12}$ and, ii) the product code structure consisting of LDPC/ CRC and systematic RS code. ${ }^{10}$ These coding schemes are used to provide UEP to the bitstream divided into packets of constant size. 


\subsubsection{LDPC Codes}

LDPC codes are a class of block codes which provide near-capacity performance while simultaneously admitting an implementable decoder. LDPC codes were first proposed by Gallager in his $1960 \mathrm{PhD}$. dissertation at MIT ${ }^{8}$ and was scarcely considered in many years that followed due to heavy hardware requirements. The study of LDPC codes was resurrected in the mid-1990's with the work of Mackay. ${ }^{9}$ LDPC codes have a very sparse parity check matrix $H$ (i.e., very few 1's and mostly 0 's) and it is generated by applying random perturbations to the zero matrix until a specified number of ones appear in each column and roughly fixed equal number of ones appear in each row. Such LDPC codes are called regular LDPC codes. The associated generator matrix $G$ is obtained by Gaussian elimination of $H$. The sparseness of $H$ eliminates the need for interleavers at both encoder and decoder and also, facilitates the faster decoding of such codes for even large block lengths.

The decoding of LDPC codes is done by an iterative probabilistic algorithm known as the belief-propagation or sum-product algorithm. This algorithm is better understood with the help of Tanner graphs. ${ }^{16}$ It starts with some initial probabilities of code bits and iteratively updates these probabilities based on message-passing. It performs parity checks until all the parity checks are satisfied or a predetermined maximum number of iterations are done. The decision is then taken on all the received bits based on the final probabilities values.

For both the proposed channel coding schemes, constant rate regular LDPC codes are used.

\subsubsection{RCPC Codes}

In the first coding scheme mentioned above, RCPC $\operatorname{codes}^{7}$ are used to provide the UEP to the bitstream. RCPC codes form a class of convolutional codes that are obtained by puncturing the output of a "mother" convolutional code. Convolutional coding is accomplished by convolving the source data with a convolutional matrix $G$. In essence, rather than having a number of channel code symbols for a corresponding block of source symbols as in linear block codes, convolutional coding generates one codeword for the entire source data. The rate of a convolutional code is defined as $k / n$ where $k$ is the number of input bits and $n$ is the number of output bits. Puncturing is the process of deleting bits from the output sequence in a predefined manner so that fewer bits are transmitted than in the original coder leading to a higher coding rate. The idea of puncturing was extended to include the concept of rate compatibility. ${ }^{7}$ Rate compatibility requires that a higher rate code be a subset of a lower rate code, or that lower protection codes be embedded into higher protection codes. This is accomplished by puncturing a "mother" code of rate $1 / n$ to achieve higher rates. At the receiver, RCPC codes are decoded using the Viterbi algorithm which is a maximum-likelihood sequence estimation technique.

\subsubsection{RS Codes}

Reed Solomon ${ }^{10}$ codes are a subset of $\mathrm{BCH}^{11}$ codes and are linear block codes. A Reed-Solomon code is specified as $R S(n, k)$ with s-bit symbols. This means that the encoder takes $k$ data symbols of $s$ bits each and adds parity symbols to make an $n$ symbol codeword. There are $n-k$ parity symbols of $s$ bits each. A Reed-Solomon decoder can correct up to $t$ errors and $2 t$ erasures, where $2 t=n-k$. Given a symbol size s, the maximum codeword length $(n)$ for a Reed-Solomon code is $n=2^{s}-1$. For example, the maximum length of a code with 8-bit symbols $(s=8)$ is 255 bytes. Reed-Solomon codes may also be shortened by (conceptually) making a number of data symbols zero at the encoder, not transmitting them, and then re-inserting them at the decoder.

Reed Solomon codes are based on finite fields, often called Galois fields. A finite field has the property that arithmetic operations $(+,-, \mathrm{x}, /$ etc.) on field elements always have a result in the same field. A Reed-Solomon encoder or decoder needs to carry out these arithmetic operations. In this work, erasure-correction systematic Reed-Solomon (RS) codes are used for the second proposed channel coding scheme.

\subsubsection{CRC Codes}

CRC codes are used in this work for error detection. Details on CRC codes can be obtained from Ref. 12. 


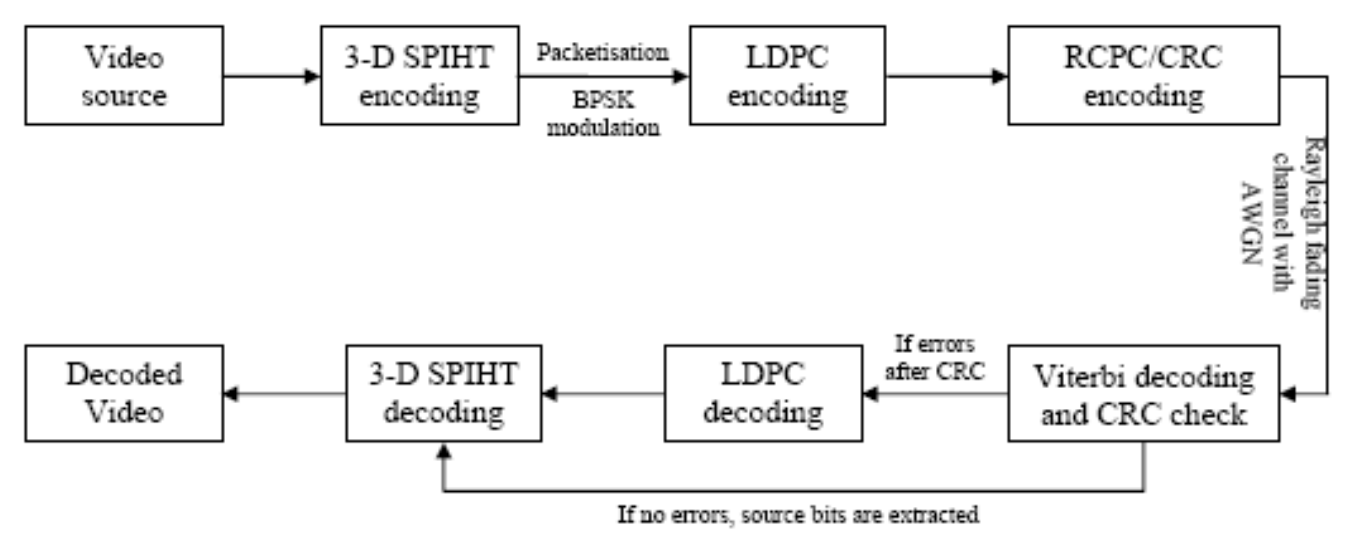

Figure 1. Block diagram of system employing concatenated LDPC+RCPC/CRC coding scheme

\section{WIRELESS VIDEO TRANSMISSION USING LDPC+RCPC/CRC CONCATENATED CODE}

\subsection{System Setup}

This scheme employs the serial concatenation of constant rate LDPC code and RCPC/CRC code for unequal error protection (UEP) of the video bitstream. In Fig. 1 we show the block diagram of this setup. The 3-D SPIHT bitstream for each group of frames (GOF) is partitioned in to "fixed-length" packets. At the receiver, if after Viterbi and CRC decoding, the packet is found to be corrupted, LDPC decoding is done to recover the data else, due to the systematic form of the LDPC codes, the source bits are immediately extracted. This reduces the overall complexity of the system.

\subsection{Optimal Resource Allocation}

Objective: Given an overall bit rate $R_{\text {budget }}$, the aim is to distribute the bits between source and channel coding such that the overall mean square distortion $D_{s+c}$ (mean square error (MSE)) is minimized or on the other hand, the overall peak signal-to-noise ratio (PSNR) is maximized $\left(P S N R=10 \log _{10}\left(255^{2} / M S E\right)\right)$. This is denoted by the following equations:

$$
\min D_{s+c} \text { subject to } R_{s+c} \leq R_{\text {budget }}
$$

and

$$
\max P_{s+c} \text { subject to } R_{s+c} \leq R_{\text {budget }}
$$

The $R_{s+c}$ term symbolizes the total bit rate used by source and channel coding for all (constant size) packets and the $D_{s+c}$ is the resulting distortion for both channel and source coding and it depends on both the channel and the encoder-decoder configuration. On the other hand, $P_{s+c}$ is the resulting video quality and is inversely proportional to the distortion. To be more specific, the distortion caused by the source coding is due to quantization and is deterministic. The distortion due to channel errors is stochastic. Therefore, the total distortion is also stochastic and the expected value of it is used, which is given as:

$$
E_{N}\left(D_{s+c}\right)=\sum_{i=0}^{N} P_{i}(R) D_{i}(R)
$$

where $N$ is the total number of packets,

$R$ is the rate allocation vector, $\left(r v_{1}, r v_{2}, \ldots, r v_{N}\right)$, which assigns to each packet $i, i=1,2, \ldots, N$, a channel code 
rate $r v_{i}$. Each of these channel code rates are chosen from a set $R V$ of $m$ channel code rates $\left(r_{1}<r_{2}<\ldots<r_{m}\right)$ used by the combination of LDPC and RCPC,

$D_{0}(R)$ is the distortion when none of the packets are received error free and is equal to the source variance, and for $i \geq 1, D_{i}(R)$ is the reconstruction distortion using the first $i$ packets,

For $i=1, \ldots, N-1, P_{i}(R)=\left(\prod_{j=1}^{i}\left(1-p\left(r v_{j}\right)\right)\right) p\left(r v_{i+1}\right)$, is the probability of no errors in the first $i$ packets but with an error in the next one, $P_{0}(R)=p\left(r v_{1}\right)$ is the probability of an error in the first packet, and $P_{N}(R)=\prod_{j=1}^{N}\left(1-p\left(r v_{j}\right)\right)$ is the probability that all the $N$ packets are correctly received.

For $i=1,2, \ldots, m, p\left(r_{i}\right)$ is the probability of a decoding error in a packet protected by the channel code rate $r_{i}$ and $p\left(r_{i}\right)=1-(1-B E R)^{l}$, where $l$ is the packet size and $B E R$ is the bit error rate after channel decoding.

As discussed above, the problem of efficient UEP and hence the optimal bit allocation for this scheme is formulated as a constrained optimization problem and is then converted into an unconstrained one by using the Lagrangian optimization, ${ }^{13-15}$ i.e.,

$$
\min J(\lambda)=D_{s+c}+\lambda R_{s+c}
$$

where $\lambda$ is the Lagrange multiplier.

The optimization works on minimizing $J$ and hence the overall expected distortion (MSE) for a given channel condition. This is accomplished by efficiently allocating the channel coding rates (from the set $R V$ of channel code rates) to the packets to meet a target transmission rate, $R_{\text {budget }}$. The rate-distortion (R-D) operating points are found by allocating combinations of channel codes across the source packets (protection strictly descending in nature) and using Eq. (4). Then the correct Lagrangian multiplier, $\lambda$ is found (using the bisection algorithm) to get the optimal operating R-D point lying on the convex hull of the overall R-D characteristic plot.

\section{WIRELESS VIDEO TRANSMISSION USING LDPC/CRC+RS PRODUCT CODE}

\subsection{Setup}

This method employs a product code structure which can be described as a two-dimensional code constructed by encoding a rectangular array of information digits with one code along rows and with another code along columns. In the product code used here, the row code is a concatenated code consisting of an outer code, CRC and an inner LDPC code while the column code is an erasure correction systematic shortened RS code. The structure of the product code is depicted in Fig. 2. A different amount of protection, provided by RS codes, is first allocated to each portion of the stream. All packets are subsequently protected using concatenated LDPC/CRC code of the constant code rate.

As in the previous channel coding method, here also the 3-D SPIHT encoded data for each group of frames (GOF) is partitioned in to "fixed-length" packets. The group of packets termed as a 'block' is protected by the product code structure described in Fig. 2. At the receiver, row-wise LDPC decoding is not needed when CRC detects the received packet to be error free. Otherwise, LDPC decoding is performed to recover the source bits and in case LDPC decoding also fails, the packet is marked as 'erased'. After receiving all the packets (for that block), the column-wise RS decoding is performed. The block diagram of this setup is as shown in Fig. 3. Each block protected with $(n, k)$ RS code can handle up to ' $\mathrm{n}-\mathrm{k}$ ' such erasures and hence can decode the source bytes correctly. However, this introduces an extra delay (as compared to the previous method) as now the decoder has to wait for the entire block to arrive before RS decoding is performed.

\subsection{Optimal resource allocation}

Again, the objective here remains same, i.e., to optimally allocate the resources and to minimize the average distortion (MSE) under a transmission rate constraint and known channel conditions, as given in Eqs. (2) and $(3)$.

Let us denote $P(n)$ as the probability that exactly $n$ rows, out the $N$ rows in a block, are 'erased' during transmission.

$$
P(n)=\left(\begin{array}{l}
N \\
n
\end{array}\right) p^{n}(1-p)^{N-n}
$$




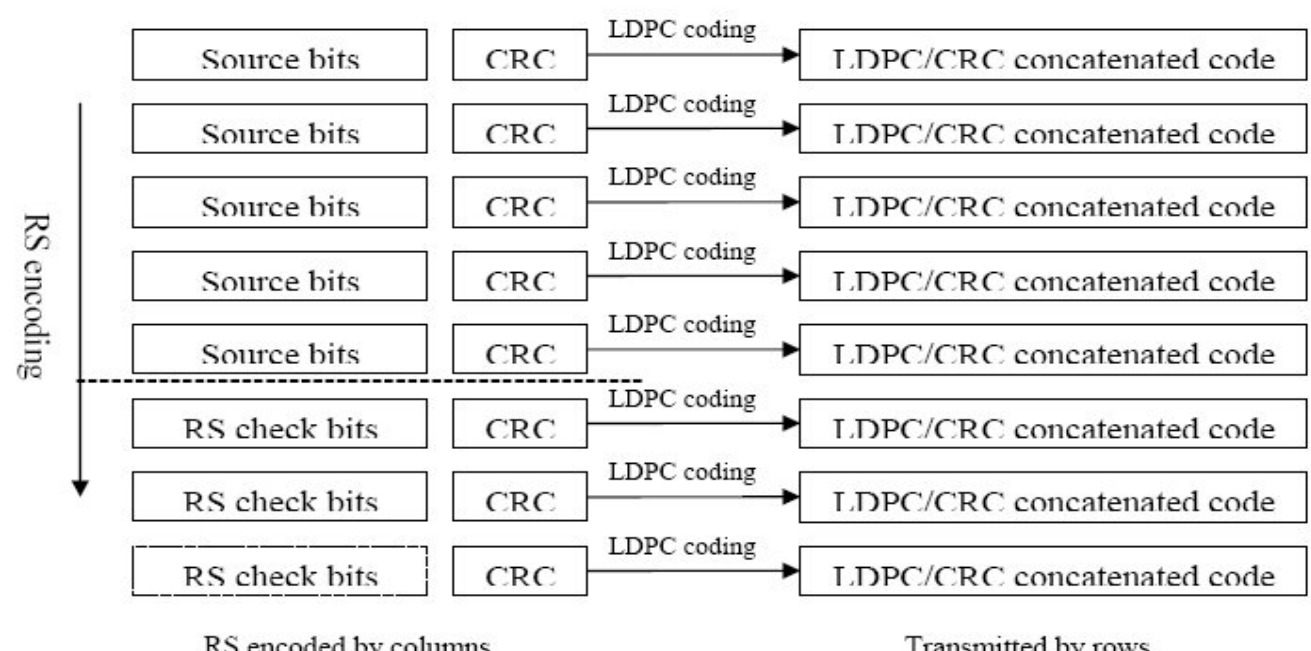

Figure 2. Schematic diagram of LDPC/CRC and RS product code

where $p$ is the probability of a row being entirely discarded due to corruption and LDPC decoding failure.

Since RS codes are used, the probability that the $k^{\text {th }}$ block is erased is equal to the probability that the number of erased rows is greater than the number of RS symbols in each column of the block:

$$
P_{L}(k)=\sum_{i=Q(k)+1}^{N} P(i)
$$

where $Q(k)$ denotes the number of RS symbols in the $k^{\text {th }}$ block. We shall consider the total rate of a block as $R_{b l o c k}$ and total rate budget, $R_{s+c}=M$. $R_{\text {block}}$, where $M$ denotes the total number of blocks to be transmitted. Here also, we assume that whenever a block is plagued by uncorrectable errors, all subsequent blocks are rendered useless and do not further change the resulting distortion. Suppose that $n$ rows are lost, then the expected distortion is given as:

$$
\begin{aligned}
& E_{M}\left(D_{s+c}\right)=D_{0} P(n>Q(1))+D_{1} P(n>Q(2), n \leq Q(1))+\ldots \\
& \quad+\ldots+D_{k} P(n>Q(k+1), n \leq Q(k))+\ldots+D_{M} P(n \leq Q(M))
\end{aligned}
$$

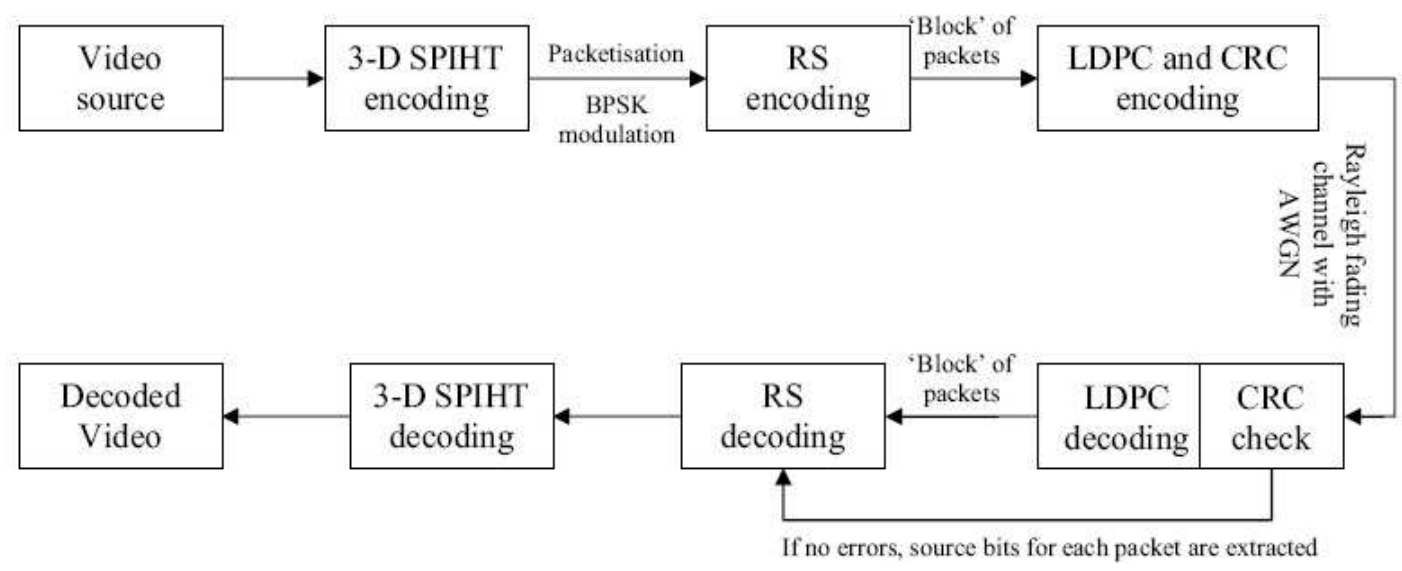

Figure 3. Block diagram of system employing product code using LDPC/CRC and RS coding scheme 
where $D_{0}$ is the distortion when none of the blocks are received error free and is equal to the source variance, and for $k \geq 1, D_{k}$ is the reconstruction distortion using the first $k$ blocks. Due to the strictly descending RS protection across the blocks, the probability that all the blocks are decodable is equal to the probability that the last block is correctly decoded. Also,

$$
P(n>Q(k), n \leq Q(k-1))=P_{L}(k)-P_{L}(k-1)
$$

Similar to the scheme mentioned in the previous section, the problem of the optimal resource allocation is formulated as a constrained optimization problem and is then converted into an unconstrained one by using Lagrangian optimization, as given by Eq. (5).

\section{EXPERIMENTAL RESULTS}

Following Figs. 1 and 3, a color "Foreman" sequence (QCIF format) is encoded using 3-D SPIHT codec at the rate of $760 \mathrm{kbps}$. The sequence consists of 300 frames, each of size $176 \times 144$ and is encoded on GOF-by-GOF basis at 30 frames/sec, i.e., each group of 16 frames is encoded and transmitted independently. The bitstream corresponding to each GOF is packetised into packets of equal length of 4110 bits. Following is the individual discussion of the results for both the proposed schemes.

\section{1. $\mathrm{LDPC}+\mathrm{RCPC} / \mathrm{CRC}$ code scheme}

The operational R-D data for the source are found by decoding the source bitstream packet-by-packet. This statistics is later used to find the expected distortion at receiver using Eq. (4). For channel coding, LDPC code of constant rate $=1 / 2$ or $2 / 3$, RCPC code of rates $(8 / 9,8 / 10,8 / 16$ and $8 / 24)$ and 16 -bit CRC code are used. The puncturing tables and other configurations for these RCPC code rates are taken from Ref. 7. The performance of the system is evaluated at three different channel SNR values, i.e., 5, 10 and $15 \mathrm{~dB}$. At the receiver, if needed, LDPC decoding is performed with maximum of 500 iterations. All the results obtained here are computed on GOF-by-GOF basis and averaged over the GOFs.

Fig. 4 shows all the operating R-D points and the curve joining the optimal PSNR points for wireless video transmission with channel $\mathrm{SNR}=5 \mathrm{~dB}$. We can see that the optimal points lie on the convex hull of the R$\mathrm{D}$ curve. Also, some of the optimal points are missed in the output of the optimization as the Lagrangian method always chooses the points lying on the convex hull. Fig. 5 displays the comparison between the PSNR optimization curves obtained at channel $\mathrm{SNR}=5,10$ and $15 \mathrm{~dB}$ and constant rate $\mathrm{LDPC}=1 / 2$. The comparison clearly shows the improvement in the performance of the system as the channel improves, i.e., as the channel improves, higher PSNR is obtained for the same transmission rate. A similar comparison is shown in Fig. 6 for constant rate $\mathrm{LDPC}=2 / 3$ rate. Fig. 7 compare the performance and optimization curves obtained from this scheme of concatenated LDPC $+\mathrm{RCPC} / \mathrm{CRC}$ code and the classical scheme of RCPC/CRC code, respectively at $\mathrm{SNR}=15 \mathrm{~dB}$ and $\mathrm{LDPC}=1 / 2$. It is clearly evident that our scheme significantly outperforms the classical scheme for the same overall channel protection and at a given transmission rate.

\section{2. $\mathrm{LDPC} / \mathrm{CRC}+\mathrm{RS}$ code scheme}

Similar to the previous case, the operational R-D data for the source are found by decoding the same bitstream block-by-block and are used to find the expected distortion at receiver using Eq. (8). For each block, 16-bit CRC is generated and appended to each packet and LDPC code of constant rate $2 / 3$ is used. Variable RS code of rates $(10,5),(15,10),(20,15),(25,15),(30,15)$ are used. In this case too, all the results obtained are computed on GOF-by-GOF basis and averaged over the GOFs.

Fig. 8 shows all the operating R-D points and the curve joining the optimal points for PSNR for video transmission over wireless channel using the product code. Fig. 9 displays the optimization curves for PSNR resulted from the simulations for channel $\mathrm{SNR}=7 \mathrm{~dB}$ and constant rate $\mathrm{LDPC}=2 / 3$. Fig. 10 compares the performances and optimization curves obtained from this scheme of LDPC/CRC+RS product code and the $\mathrm{LDPC}+\mathrm{RCPC} / \mathrm{CRC}$ code scheme. It is evident that product code structure is better than the serially concatenated codes because of the erasure correction capabilities of the RS codes. The only trade-off here is the extra delay introduced in the product code scheme. 


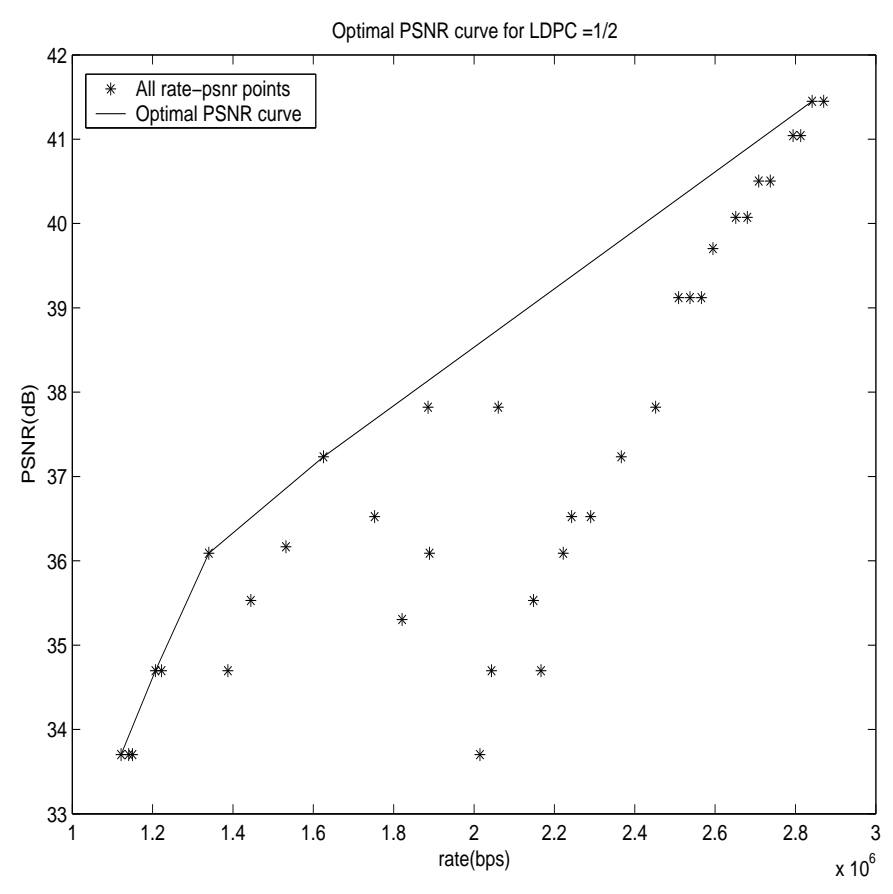

Figure 4. R-D operating points and the optimal curve for PSNR at LDPC $=1 / 2$ rate and channel $\mathrm{SNR}=5 \mathrm{~dB}$.

\section{CONCLUSIONS}

In this work, the performance of the fully scalable video coding using 3-D SPIHT codec and transmission over flat-fading Rayleigh wireless channels was developed and studied. Two different channel coding schemes, i.e., concatenated LDPC $+\mathrm{RCPC} / \mathrm{CRC}$ code and $\mathrm{LDPC} / \mathrm{CRC}+\mathrm{RS}$ product code, were developed and their performance were compared against classical scheme employing RCPC/CRC. A rate-distortion optimization method using Lagrangian multiplier was developed and carried out for the choice of number of packets (i.e., source rate) to be transmitted and channel coding rates. It was shown that the two proposed schemes outperform the classical scheme for same overall channel protection and transmission rates. Also, it was made evident that product code structure is better than the serially concatenated codes because of the erasure correction capabilities of the RS codes. The only trade-off here is the delay introduced in the former method. The rate-distortion optimization for both the schemes was also performed under different channel conditions and the results showed a significant improvement in the performance as the channel improves.

\section{REFERENCES}

1. Z. Xiong, B. Kim and W.A. Pearlman, "Progressive video coding for noisy channels", Proceedings of International Conference on Image Processing, Volume:1, pp. 334-337, October 1998.

2. P.G. Sherwood and K. Zeger, "Error protection for progressive image transmission over memoryless and fading channels", IEEE Transactions on Communications, Volume:46, Issue:12, pp. 1555-1559, December 1998.

3. D.G. Sachs, R. Anand and K. Ramchandran, "Wireless image transmission using multiple-description based concatenated codes", Data Compression Conference Proceedings, March 2000.

4. V. Chande and N. Farvardin, "Progressive transmission of images over memoryless noisy channels", IEEE Journal on Selected Areas in Communications, Volume:18, Issue:6, June 2000.

5. A. Said and W.A. Pearlman, "A new fast, and efficient image codec based on set partitioning in hierarchical trees", IEEE Transactions on Circuits and Systems for Video Technology, Volume:6, Issue:3, pp. 243-250, June 1996. 


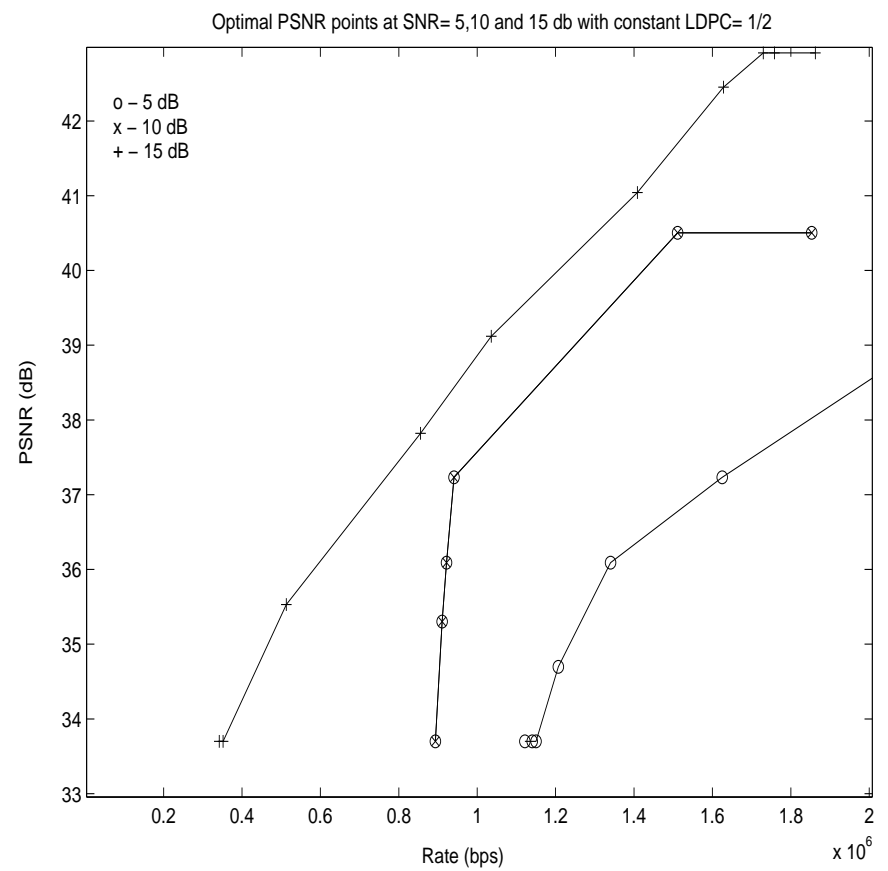

Figure 5. Comparison of R-D optimal curves for PSNR at $\mathrm{LDPC}=1 / 2$ rate at channel $\mathrm{SNR}=5,10$ and $15 \mathrm{~dB}$.

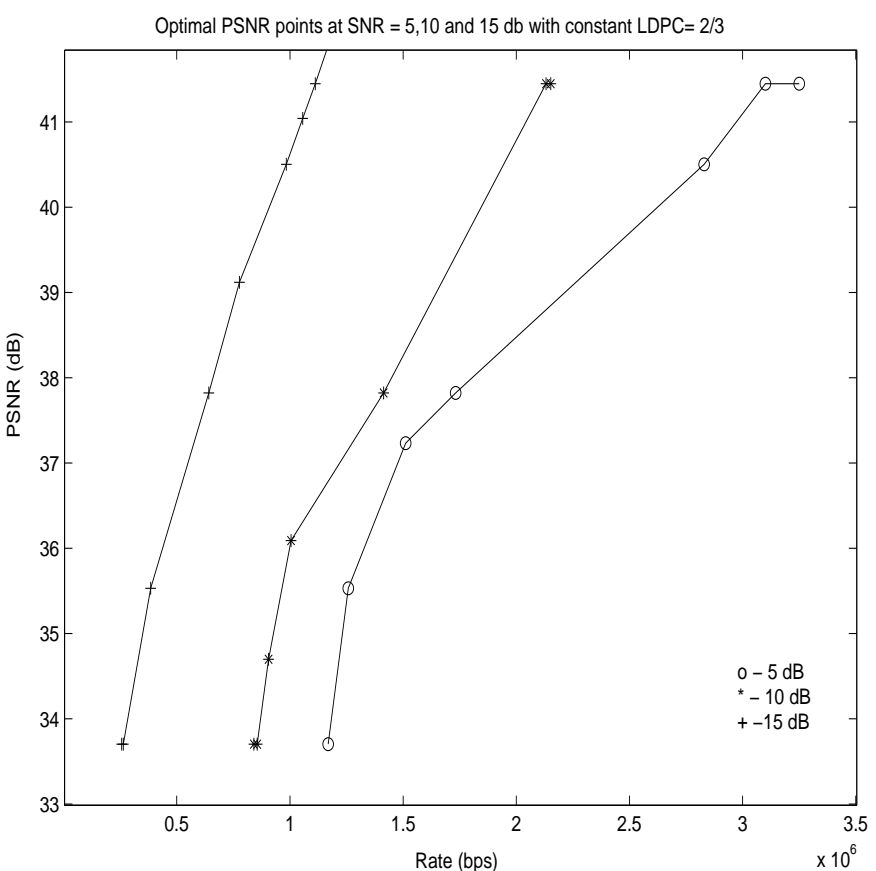

Figure 6. Comparison of R-D optimal curves for PSNR at LDPC $=2 / 3$ rate at channel SNR=5,10 and $15 \mathrm{~dB}$. 


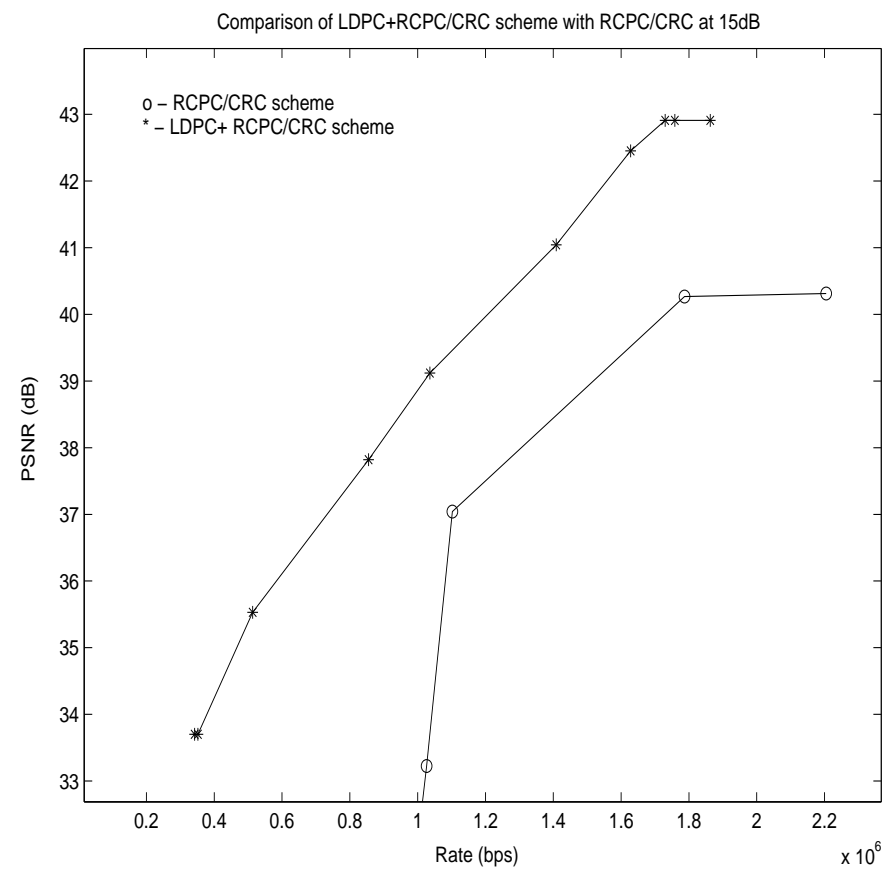

Figure 7. Comparison of R-D optimal curves for PSNR between LDPC+RCPC/CRC scheme and RCPC/CRC scheme.

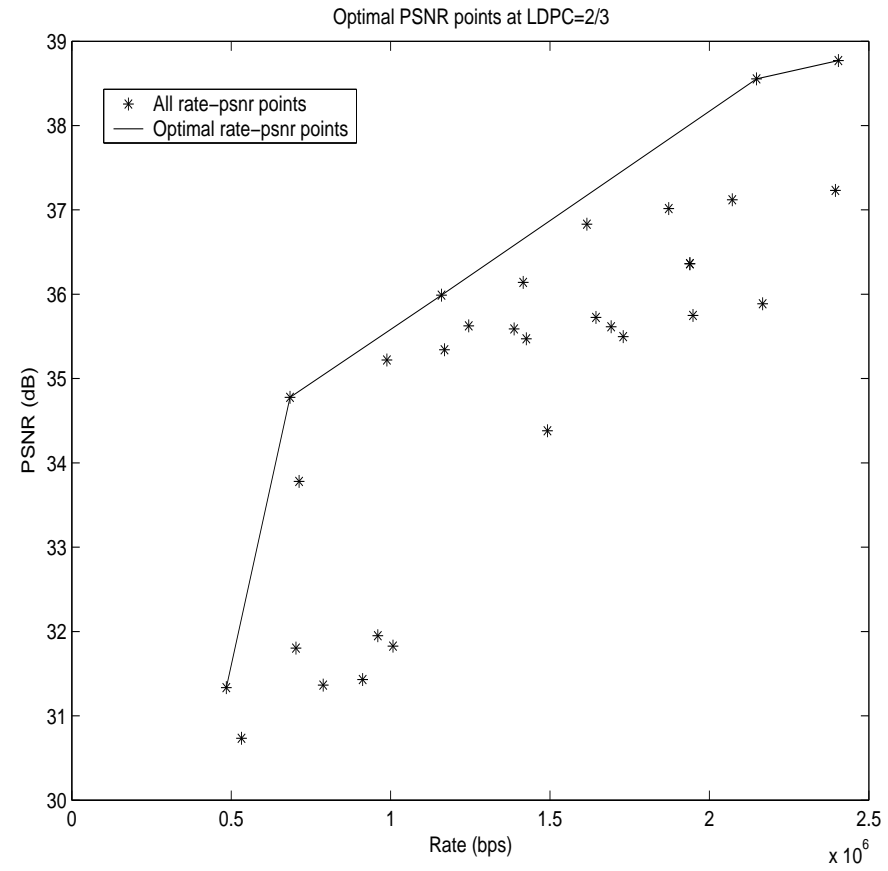

Figure 8. Rate-Distortion operating points and the optimal curve for PSNR using the product code 


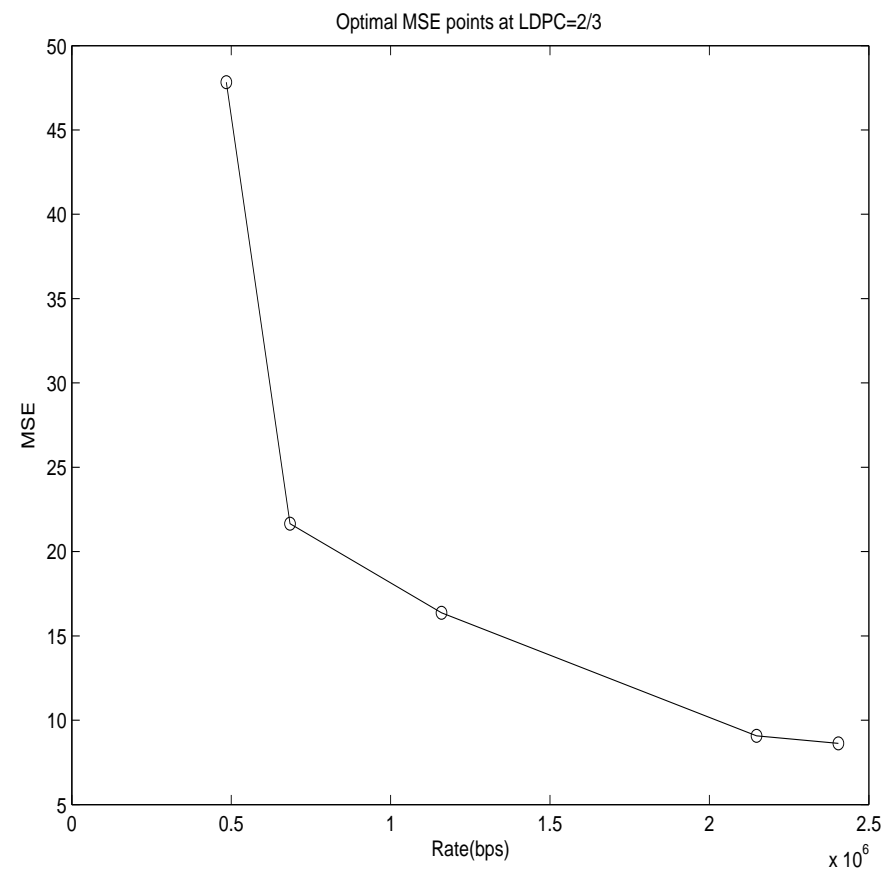

Figure 9. Rate-Distortion optimization curve for PSNR using the product code at channel $\mathrm{SNR}=7 \mathrm{~dB}$ and $\mathrm{LDPC}=2 / 3$

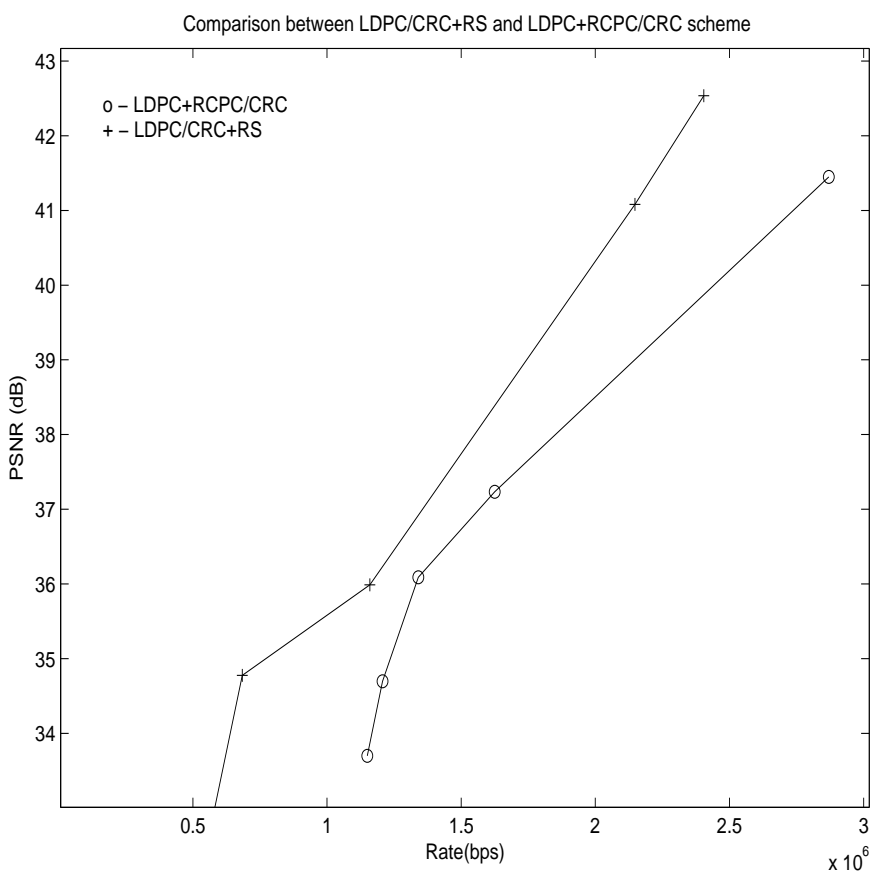

Figure 10. Comparison between LDPC/CRC+RS and LDPC + RCPC/CRC scheme in terms of PSNR 
6. B. Kim, Z. Xiong and W.A. Pearlman, "Very low bit-rate scalable video coding with 3-D set partitioning in hierarchical trees (3-D SPIHT)", IEEE Transactions on Circuits and Systems for Video Technology, Volume:10, Issue:8, December 2000.

7. J. Hagenauer, "Rate compatible punctured convolutional codes (RCPC codes) and their applications", IEEE Transactions on Communications, Volume 36, pp. 389-399, April 1988.

8. R.G. Gallager, "Low density parity check codes", IEEE Transactions on Information Theory, Volume IT-8, pp. 2128, January 1962.

9. D.J.C. Mackay, "Good error-correcting codes based on very sparse matrices", IEEE Transactions on Information Theory, pp. 543-547, March 1999.

10. I.S. Reed and G. Solomon, "Polynomial codes over certain certain finite fields", Journal of the Society for Industrial and Applied mathematics, 1960.

11. S. Liu and Jr. D.J. Costello, "Error Control Coding: Fundamentals and Applications", Prentice Hall, Englewood Cliffs, NJ, 1983.

12. W.H. Press, B.P. Flannery, S.A. Teukolsky and W.T. Vetterling, "Cyclic redundancy and other checksums", Ch. 20.3 in Numerical Recipes in FORTRAN: The Art of Scientific Computing, 2nd ed. Cambridge, England: Cambridge University Press, pp. 888-895, 1992.

13. A. Ortega and K. Ramchandran, "Rate distortion methods for image and video compression", IEEE Signal Processing Magazine, Volume:15, Issue:15, pp. 23-50, November 1998.

14. L.P. Kondi, F. Ishtiaq, A.K. Katsaggelos, "Joint source-channel coding for motion-compensated DCT-based SNR scalable video", IEEE Transactions on Image Processing, Volume:11, pp. 1043-1052., September 2002.

15. L.P. Kondi, D. Srinivasan, D.A. Pados, S.N. Batalama, "Layered video transmission over multirate DSCDMA wireless systems", IEEE Transactions on Circuits and Systems for Video Technology, under review.

16. R.M. Tanner, "A recursive approach to low complexity codes", IEEE Transactions on Information Theory, pp. 399-431, September 1981.

17. G. Partasides, L.P. Kondi, "Scalable video transmission over orthogonal frequency division multiplexing wireless channels", Proceedings IEEE Canadian Conference on Electrical and Computer Engineering, Niagara Falls, Canada, May 2004. 